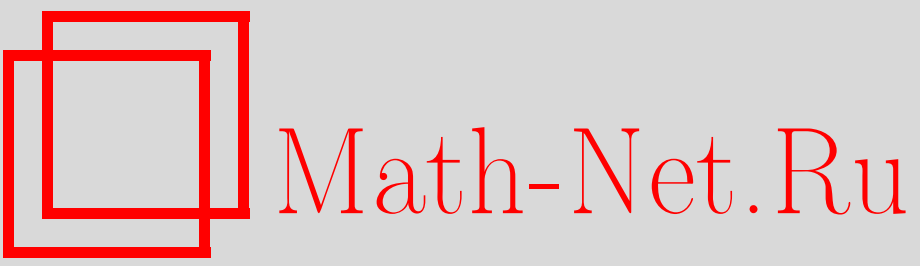

С. В. Козырев, $p$-адические псевдодифференциальные операторы и $p$-адические всплески, ТМФ, 2004, том 138, номер 3, 383-394

DOI: https://doi.org/10.4213/tmf31

Использование Общероссийского математического портала Math-Net.Ru подразумевает, что вы прочитали и согласны с пользовательским соглашением

http://www.mathnet.ru/rus/agreement

Параметры загрузки:

IP : 54.224 .135 .184

26 апреля 2023 г., $17: 29: 30$ 
ТЕОРЕТИЧЕСКАЯ

И МАТЕМАТИЧЕСКАЯ

ФИЗИКА

Том 138, № 3

март, 2004

(C) 2004 г.

С.В. Козырев*

\section{p-АДИЧЕСКИЕ ПСЕВДОДИФФЕРЕНЦИАЛЬНЫЕ ОПЕРАТОРЫ И $p$-АДИЧЕСКИЕ ВСПЛЕСКИ}

Вводится новый широкий класс $p$-адических псевдодифференциальных операторов. Показано, что базис $p$-адических всплесков является базисом из собственных векторов для введенных операторов.

Ключевые слова: $p$-адическая диффузия.

\section{1. ВВЕДЕНИЕ}

В настоящей работе вводится семейство генераторов $p$-адических псевдодифференциальных операторов, являюшихся генераторами $p$-адических диффузий. Построенные генераторы, в отличие от оператора Владимирова $p$-адического дробного дифференцирования [1], будут приводиться к диагональному виду не преобразованием Фурье, а в базисе $p$-адических всплесков, введенных в работе [2]. Поэтому будем называть такие операторы трансляционно-неинвариантными (т.е. неперестановочными с операторами сдвигов).

Далее рассматривается только вопрос существования указанных операторов. Чтобы показать, что введенные операторы $T$ порождают диффузии, надо убедиться, что соответствуюшие эволюции $e^{-t T}$ сушествуют, сохраняют конус положительных функций и полное число частиц. На формальном уровне эти свойства имеют место, строгое доказательство будет рассмотрено в другой публикации.

Ультраметрические диффузии рассматривались в работах [1], [3]-[6], а также в статьях [7]-[9] при описании процессов релаксации в неупорядоченных системах, в частности, в спиновых стеклах и белках. Ниже описываются более обшие генераторы диффузий.

Поле $p$-адических чисел является простейшим примером ультраметрического пространства. В работах [2]-[16] изучаются модели $p$-адической математической физики. В работах $[7],[14] p$-адический анализ применялся к описанию нарушения репличной симметрии (обсуждение метода реплик см. в [17]). Было показано, что матрица Паризи, описываюшая нарушение репличной симметрии (до предельного перехода $n \rightarrow 0$ ), есть дискретный аналог $p$-адического псевдодифференциального оператора. В данной работе вводится семейство генераторов ультраметрических диффузий. Дискретные аналоги

\footnotetext{
${ }^{*}$ Институт химической физики РАН, Москва, Россия. E-mail: kozyrev@mi.ras.ru
} 
генераторов этих диффузий будут служить новыми примерами репличных матриц. Интересной задачей является приложение этих матриц к неупорядоченным моделям (при этом необходимо выполнить предел $n \rightarrow 0$ для введенных операторов). Другим возможным применением развиваемого далее подхода является его приложение к изучению случайных блужданий на иерархических ландшафтах энергии, исследование явлений релаксации в сложных системах и так называемой “кинетики из бассейна в бассейн” ("basin to basin kinetics") (см. [18]-[22]).

В разделе 2 приведены некоторые сведения из $p$-адического анализа и описан базис $p$-адических всплесков. В разделе 3 вводится семейство генераторов ультраметрических диффузий и доказывается, что эти генераторы диагональны в базисе $p$-адических всплесков. В разделе 4 обсуждаются некоторые примеры генераторов ультраметрической диффузии и вычисляется асимптотика заселенности единичного диска для ультраметрической диффузии. В разделе 5 рассмотрены возможные физические применения результатов данной работы.

\section{2. $p$-АДИЧЕСКИЙ АНАЛИЗ}

Ультраметрическое пространство - это метрическое пространство с метрикой $\|\cdot\|$, удовлетворяюшей сильному неравенству треугольника

$$
\|x y\| \leqslant \max (\|x z\|,\|y z\|), \quad \forall z .
$$

Простейшим примером ультраметрического пространства является поле $Q_{p} p$-адических чисел. Поле $Q_{p}$ является пополнением поля рациональных чисел $Q$ по $p$-адической норме на $Q$. Эта норма определяется следуюшим образом. Произвольное рациональное число $x$ может быть записано в виде $x=p^{\gamma} m / n$, где $m$ и $n$ не делятся на $p$. $p$-Адическая норма рационального числа $x=p^{\gamma} m / n$ есть $|x|_{p}=p^{-\gamma}$. Сильное неравенство треугольника для $p$-адических чисел принимает вид

$$
|x+y|_{p} \leqslant \max \left(|x|_{p},|y|_{p}\right) .
$$

$p$-Адические числа находятся во взаимно-однозначном соответствии с (сходяшимися в $p$-адической норме) рядами

$$
x=\sum_{\gamma=\gamma_{0}}^{\infty} x_{i} p^{i}, \quad x_{i}=0, \ldots, p-1 .
$$

Кольцо $Z_{p}$ целых $p$-адических чисел содержит $p$-адические числа вида

$$
x=\sum_{\gamma=0}^{\infty} x_{i} p^{i}, \quad x_{i}=0, \ldots, p-1 .
$$

Мы отождествляем группу $Q_{p} / Z_{p}$ со множеством дробей вида

$$
x=\sum_{\gamma=\gamma_{0}}^{-1} x_{i} p^{i}, \quad x_{i}=0, \ldots, p-1 .
$$


Оператор Владимирова $p$-адического дробного дифференширования определяется следуюшим образом [1]:

$$
D^{\alpha} f(x)=\frac{1}{\Gamma_{p}(-\alpha)} \int_{Q_{p}} \frac{f(x)-f(y)}{|x-y|_{p}^{1+\alpha}} d y,
$$

где $d y$ - мера Хаара на $Q_{p}$ и $\Gamma_{p}(-\alpha)$ - постоянная. Этот оператор диагонализуется $p$-адическим преобразованием $\Phi$ урье $F$,

$$
D^{\alpha} f(x)=F^{-1} \circ|k|_{p}^{\alpha} \circ F f(x) .
$$

p-Адические всплески введены в работе [2], где была доказана следуюшая теорема.

Теорема 1. Набор функиий $\left\{\psi_{\gamma j n}\right\}$,

$$
\psi_{\gamma j n}(x)=p^{-\frac{\gamma}{2}} \chi\left(p^{\gamma-1} j x\right) \Omega\left(\left|p^{\gamma} x-n\right|_{p}\right), \quad \gamma \in \mathbf{Z}, \quad n \in Q_{p} / Z_{p}, \quad j=1, \ldots, p-1,
$$

является ортонормированныц базисом в пространстве $L^{2}\left(Q_{p}\right)$, состоящим из собственных векторов оператора $D^{\alpha}$,

$$
D^{\alpha} \psi_{\gamma j n}=p^{\alpha(1-\gamma)} \psi_{\gamma j n}
$$

Здесь $\Omega(x)$ - индикатор отрезка $[0,1]$. Для характеристической функции $p$-адического диска получаем выражение

$$
\Omega\left(|x|_{p}\right)= \begin{cases}1, & |x|_{p} \leqslant 1 \\ 0, & |x|_{p}>1\end{cases}
$$

Функция $\chi(x)$ является комплекснозначным характером $p$-адического аргумента, который для числа $x$, заданного рядом (1), определен как

$$
\chi(x)=\exp \left(2 \pi i \sum_{\gamma=\gamma_{0}}^{-1} x_{i} p^{i}\right) .
$$

Теорема 1 показывает, что $p$-адические всплески образуют базис собственных векторов оператора (2), который рассматривался как генератор ультраметрической дифофузии (см. [1], [7]-[9]). В данной работе мы вводим более обший класс таких генераторов. Эти генераторы не могут быть диагонализованы $p$-адическим преобразованием Фурье. Тем не менее мы покажем, что они все еше диагонализуемы $p$-адическим вейвлет-преобразованием (т.е. $p$-адические всплески являются собственными векторами генераторов).

$p$-Адические числа связаны с деревьями Брюа-Титса [23]. Дерево Брюа-Титса это бесконечное дерево (с бесконечным числом вершин и ребер), в котором каждая вершина связана в точности с $p+1$ другими вершинами. $p$-А дическая проективная прямая, содержашая поле $p$-адических чисел плюс одну бесконечную точку, представляется как пространство классов эквивалентности бесконечно продолженных путей в дереве Брюа-Титса. Два пути эквивалентны, если они совпадают начиная с некоторой

2 Теоретическая и математическая физика, т. 138, № 3, 2004 г. 
точки. В частности, пространство бесконечно продолженных путей, проходящих через фиксированную вершину и через $p$ ребер, примыкаюших к вершине, гомеоморфно $p$-адическому диску. Эта конструкция является аналогом модели Пуанкаре плоскости Лобачевского, где дерево Брюа-Титса представляет собой аналог плоскости Лобачевского, $p$-адическая (проективная) прямая - аналог абсолюта, и (единственный) путь в дереве Брюа-Титса, соединяющий $x$ и $y$ в поле $Q_{p},-$ аналог геодезической, соединяющей точки абсолюта.

Введем уровень, или высоту, вершины следуюшим образом. Сопоставим каждой вершине в дереве Брюа-Титса $p$-адический диск, соответствующий множеству полубесконечных путей, начинаюшихся в вершине. Это множество содержит все пути, проходяшие через $p$ из $p+1$ ребер, примыкаюших к вершине (исключая одно ребро, лежашее на пути, проходящем через бесконечную точку абсолюта). Мы называем уровнем вершины $\log _{p} r$, где $r$ - радиус этого диска. Для двух точек $x, y$ в $Q_{p}$ сушествует единственный путь в дереве Брюа-Титса, соединяюший эти точки. Этот путь содержит единственную точку $A(x, y)$ с наибольшим уровнем.

\section{3. ПОСТРОЕНИЕ ПСЕВДОДИФФЕРЕНЦИАЛЬНЫХ ОПЕРАТОРОВ}

В работах [24], [25] были рассмотрены примеры ультраметрических диффузий на дискретном множестве. В работах [1], [3]-[9] исследовались примеры $p$-адических диффузий. В этом разделе мы вводим семейство генераторов ультраметрических диффузий на поле $Q_{p} p$-адических чисел. Существование соответствующих дифффузий (т.е. возможность взятия экспоненты от генератора) обсуждаться в данной работе не будет. Введенные псевдодифференциальные операторы, генерируюшие ультраметрические диффузии, обобшают оператор Владимирова [1].

Введем оператор

$$
T f(x)=\int T(x, y)(f(x)-f(y)) d y,
$$

где $x, y$ лежат в поле $Q_{p}$. Оператор $T$ рассматривается как оператор в пространстве $L^{2}\left(Q_{p}\right)$ комплекснозначных квадратично-интегрируемых функций $p$-адического аргумента.

В дальнейшем будут определены некоторые условия на ядро $T(x, y)$. Генератор (5), удовлетворяюший этим условиям, будем называть генератором ультраметрической диффузии. Мы покажем, что этот генератор будет диагонален в базисе $p$-адических всплесков, введенных в работе [2]. Это означает, что условие ультраметричности диффузии делает задачу исследования диффузии точно решаемой. Далее мы рассмотрим генераторы $p$-адических диффузий. Интересно обобщение на случай более обших ультраметрических пространств.

ОПРЕДЕЛЕНИЕ. Назовем вероятность перехода (и соответствуюшую диффузию с генератором (5)) ультраметрической, если вероятность перехода $T(x, y)$ зависит только от наивысшей вершины $A(x, y)$ на пути в дереве Брюа-Титса, соединяюшем $x$ и $y$.

Вероятность перехода $T(x, y)$ симметрична по $x, y$, и имеет место следуюшая лемма. 
ЛЕмма. Функиия $T(x, y)$ симметрична, положстельна и локально постоянна по у вне некоторой окрестности $x$. Более того, для фиксированного $x$ выполнено следующее условие:

$$
T(x, y)=\text { const }, \quad \text { ecлu } \quad|x-y|_{p}=\text { const } .
$$

Обратно, если условие (6) выполнено, функция T( $x, y)$ удовлетворяет условиям данного выше определения.

Легко видеть, что оператор Владимирова и схожие операторы с трансляционно-инвариантными ядрами $T(x, y)$ (которые зависят только от разности $(x-y)$ и, следовательно, при соответствующих условиях интегрируемости ядра диагонализуются преобразованием Фурье) удовлетворяют условиям леммы. Далее будут приведены примеры генераторов, ядра которых не являются транслящионно-инвариантными.

Следуюшая теорема описывает общую форму ядра для генератора ультраметрической диффузии.

ТЕОРема 2. Функиия вида

$$
T(x, y)=\sum_{\gamma n} T^{(\gamma n)} \delta_{1,\left|p^{\gamma} x-p^{\gamma} y\right|_{p}} \Omega\left(\left|p^{\gamma} x-n\right|_{p}\right)
$$

где $T^{(\gamma n)} \geqslant 0, \quad \gamma \in Z, \quad n \in Q_{p} / Z_{p}$, удовлетворяет условиям леммы. Более того, произвольная функция, удовлетворяющая условию (6), может быть представлена в виде (7).

ДокАЗАТЕЛЬСтво. Положительность функции $T(x, y)$ очевидна. Докажем симметричность $T(x, y)$. Рассмотрим выражение

$$
T(x, y)-T(y, x)=\sum_{\gamma n} T^{(\gamma n)} \delta_{1,\left|p^{\gamma} x-p^{\gamma} y\right|_{p}}\left(\Omega\left(\left|p^{\gamma} x-n\right|_{p}\right)-\Omega\left(\left|p^{\gamma} y-n\right|_{p}\right)\right)
$$

и докажем, что оно тождественно равно нулю. Рассмотрим случай, когда число $x$ таково, что следующая характеристическая функция не равна нулю:

$$
\Omega\left(\left|p^{\gamma} x-n\right|_{p}\right)=1
$$

Отсюда следует, что

$$
\left|x-p^{-\gamma} n\right|_{p} \leqslant p^{\gamma}
$$

Если

$$
\delta_{1,\left|p^{\gamma} x-p^{\gamma} y\right|_{p}} \neq 0
$$

TO

$$
|x-y|_{p}=p^{\gamma} .
$$

Из формул (9), (10) и ультраметричности следует, что

$$
\Omega\left(\left|p^{\gamma} y-n\right|_{p}\right)=1
$$


Следовательно, соответствующие члены в (8) сокращаются, что доказывает симметричность $T(x, y)$.

Докажем, что функция $T(x, y)$, задаваемая формулой (7), удовлетворяет условию (6). Выберем $x \in Q_{p}$. Тогда для числа $y$, лежашего на сфере с центром в $x$ и радиусом $p^{\gamma}$, получим

$$
\delta_{1,\left|p^{\gamma} x-p^{\gamma} y\right|_{p}}=1
$$

и функция

$$
\Omega\left(\left|p^{\gamma} x-n\right|_{p}\right)
$$

будет равна нулю или единице в зависимости от того, будет ли $p^{-\gamma} n$ лежать в диске $\left\{y:|x-y|_{p} \leqslant p^{\gamma}\right\}$ или нет. Следовательно, функция $T(x, y)$ будет постоянной на рассмотренной сфере и условие (6) будет выполнено. Это доказывает, что функция $T(x, y)$, удовлетворяющая условиям теоремы 2 , будет удовлетворять условиям леммы.

Обратно, легко видеть, что ядро (7) для чисел $x, y$, лежаших в диске с центром в $p^{-\gamma} n$ радиуса $p^{\gamma}$ и удовлетворяюших условию $|x-y|_{p}=p^{\gamma}$, принимает значение $T^{(\gamma n)}$.

Поскольку все пространство $x, y \in Q_{p} \times Q_{p}$ есть несвязное объединение подмножеств такого вида, то, выбирая произвольные положительные значения $T^{(\gamma n)}$, можно построить произвольное ядро, удовлетворяющее условию (6). Это завершает доказательство теоремы.

В следующей теореме мы вычисляем собственные значения генератора ультраметрической диффузии.

ТЕОРемА 3. Пусть ядро (6) удовлетворяет условию сходимости всех интезралов вида (12) для любых $\gamma, n$. Тогда оператор (5) есть хорошо определенный оператор с плотной областью определения в пространстве $L^{2}\left(Q_{p}\right)$ и р-адические

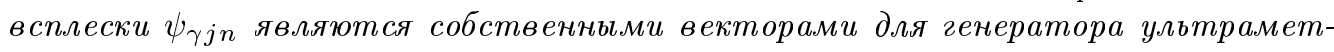
рической диффузии

$$
T \psi_{\gamma j n}=\lambda_{\gamma n} \psi_{\gamma j n}
$$

с собственными значениями

$$
\lambda_{\gamma n}=\int_{\left|n-p^{\gamma} y\right|_{p}>1} T\left(p^{-\gamma} n, y\right) d y+p^{\gamma} T\left(p^{-\gamma} n, p^{-\gamma}(n+1)\right) .
$$

ДокАЗАТЕЛЬСтво. Используем утверждение леммы. Рассмотрим всплеск $\psi_{\gamma j n}$, тогда

$$
T \psi_{\gamma j n}(x)=\int T(x, y)\left(\psi_{\gamma j n}(x)-\psi_{\gamma j n}(y)\right) d y
$$

Пусть $\left|p^{\gamma} x-n\right|_{p}>1$. Тогда из леммы следует, что

$$
T \psi_{\gamma j n}(x)=\psi_{\gamma j n}(x) \int T(x, y) d y-T(x, n) \int \psi_{\gamma j n}(y) d y=0
$$


Пусть теперь $\left|p^{\gamma} x-n\right|_{p} \leqslant 1$. Тогда опять по лемме имеем

$$
\begin{aligned}
& T \psi_{\gamma j n}(x)=\left(\int_{\left|p^{\gamma} x-p^{\gamma} y\right|_{p}>1}+\int_{\left|p^{\gamma} x-p^{\gamma} y\right|_{p}=1}+\right. \\
& \left.+\int_{\left|p^{\gamma} x-p^{\gamma} y\right|_{p}<1}\right) T(x, y)\left(\psi_{\gamma j n}(x)-\psi_{\gamma j n}(y)\right) d y= \\
& =\left(\int_{\left|p^{\gamma} x-p^{\gamma} y\right|_{p}>1}+\int_{\left|p^{\gamma} x-p^{\gamma} y\right|_{p}=1}\right) T(x, y)\left(\psi_{\gamma j n}(x)-\psi_{\gamma j n}(y)\right) d y= \\
& =\psi_{\gamma j n}(x) \int_{\left|p^{\gamma} x-p^{\gamma} y\right|_{p}>1} T(x, y) d y+ \\
& +\int_{\left|p^{\gamma} x-p^{\gamma} y\right|_{p}=1} T(x, y)\left(\psi_{\gamma j n}(x)-\psi_{\gamma j n}(y)\right) d y= \\
& =\psi_{\gamma j n}(x) \int_{\left|n-p^{\gamma} y\right|_{p}>1} T\left(p^{-\gamma} n, y\right) d y+ \\
& +\sum_{l=0}^{p-1} \int_{|z|_{p}<p^{\gamma}} T\left(x, x+p^{-\gamma} l+z\right)\left(\psi_{\gamma j n}(x)-\psi_{\gamma j n}\left(x+p^{-\gamma} l+z\right)\right) d z= \\
& =\psi_{\gamma j n}(x) \int_{\left|n-p^{\gamma} y\right|_{p}>1} T\left(p^{-\gamma} n, y\right) d y+ \\
& +p^{\gamma-1} \sum_{l=1}^{p-1} T\left(x, x+p^{-\gamma} l\right)\left(\psi_{\gamma j n}(x)-\psi_{\gamma j n}\left(x+p^{-\gamma} l\right)\right) \text {. }
\end{aligned}
$$

Последнее равенство следует из леммы и локального постоянства функции $\psi_{\gamma j n}$. Используя равенство

$\psi_{\gamma j n}\left(x+p^{-\gamma} l\right)=p^{-\gamma / 2} \chi\left(p^{\gamma-1} j\left(x+p^{-\gamma} l\right)\right) \Omega\left(\left|p^{\gamma}\left(x+p^{-\gamma} l\right)-n\right|_{p}\right)=\chi\left(p^{-1} j l\right) \psi_{\gamma j n}(x)$

и лемму, получим

$$
\begin{aligned}
T \psi_{\gamma j n}(x)= & \psi_{\gamma j n}(x)\left(\int_{\left|n-p^{\gamma} y\right|_{p}>1} T\left(p^{-\gamma} n, y\right) d y+\right. \\
& \left.+p^{\gamma-1} T\left(p^{-\gamma} n, p^{-\gamma}(n+1)\right) \sum_{l=1}^{p-1}\left(1-\chi\left(p^{-1} j l\right)\right)\right) .
\end{aligned}
$$

Поскольку

$$
\sum_{l=1}^{p-1}\left(1-\chi\left(p^{-1} j l\right)\right)=p
$$

TO

$$
T \psi_{\gamma j n}(x)=\psi_{\gamma j n}(x)\left(\int_{\left|n-p^{\gamma} y\right|_{p}>1} T\left(p^{-\gamma} n, y\right) d y+p^{\gamma} T\left(p^{-\gamma} n, p^{-\gamma}(n+1)\right)\right),
$$

что доказывает формулу (12). Следовательно, оператор $T$ хорошо определен на базисе в пространстве $L^{2}\left(Q_{p}\right)$, что завершает доказательство теоремы.

Следующее следствие дает простое представление для собственных значений генератора с ядром (7). 
СлЕДСТвИЕ. Пусть ряд

$$
\sum_{\gamma=0}^{\infty} p^{\gamma} T^{(\gamma 0)}<\infty
$$

сходится для любого $n$. Тогда оператор (5), соответствующий ядру (7), хорошо определен в пространстве $L^{2}\left(Q_{p}\right)$, диагонализуется в базисе р-адических всплесков и имеет собственные значения

$$
\lambda_{\gamma n}=p^{\gamma} T^{(\gamma n)}+\left(1-p^{-1}\right) \sum_{\gamma^{\prime}=\gamma+1}^{\infty} p^{\gamma^{\prime}} T^{\left(\gamma^{\prime}, p^{\gamma^{\prime}-\gamma} n\right)} .
$$

Отметим, что условие сходимости ряда (13) әквивалентно сходимости интеграла (12).

ДОКАЗАТЕЛЬСТво. Подставляя (7) в (12), получим

$$
\begin{aligned}
\lambda_{\gamma n}= & \int_{\left|n-p^{\gamma} y\right|_{p}>1} \sum_{\gamma^{\prime} n^{\prime}} T^{\left(\gamma^{\prime} n^{\prime}\right)} \delta_{1,\left|p^{\gamma^{\prime}} p^{-\gamma} n-p^{\gamma^{\prime}} y\right|_{p}} \Omega\left(\left|p^{\gamma^{\prime}} p^{-\gamma} n-n^{\prime}\right|_{p}\right) d y+ \\
& +p^{\gamma} \sum_{\gamma^{\prime} n^{\prime}} T^{\left(\gamma^{\prime} n^{\prime}\right)} \delta_{1,\left|p^{\gamma^{\prime}} p^{-\gamma} n-p^{\gamma^{\prime}} p^{-\gamma}(n+1)\right|_{p}} \Omega\left(\left|p^{\gamma^{\prime}} n-n^{\prime}\right|_{p}\right)= \\
= & \sum_{\gamma^{\prime} n^{\prime}} T^{\left(\gamma^{\prime} n^{\prime}\right)} p^{\gamma^{\prime}}\left(1-p^{-1}\right) \theta\left(\gamma^{\prime}-\gamma\right) \Omega\left(\left|p^{\gamma^{\prime}} p^{-\gamma} n-n^{\prime}\right|_{p}\right)+ \\
& +p^{\gamma} \sum_{n^{\prime}} T^{\left(\gamma n^{\prime}\right)} \Omega\left(\left|n-n^{\prime}\right|_{p}\right)=p^{\gamma} T^{(\gamma n)}+\left(1-p^{-1}\right) \sum_{\gamma^{\prime}=\gamma+1}^{\infty} p^{\gamma^{\prime}} T^{\left(\gamma^{\prime}, p^{\gamma^{\prime}-\gamma} n\right)}
\end{aligned}
$$

где мы использовали свойства

$$
\begin{gathered}
\int_{\left|n-p^{\gamma} y\right|_{p}>1} \delta_{1,\left|p^{\gamma^{\prime}} p^{-\gamma} n-p^{\gamma^{\prime}} y\right|_{p}} d y=p^{\gamma^{\prime}}\left(1-p^{-1}\right) \theta\left(\gamma^{\prime}-\gamma\right) \\
\theta(\gamma)= \begin{cases}0, & \gamma \leqslant 0 \\
1, & \gamma>0\end{cases} \\
\delta_{1,\left|p^{\gamma^{\prime}} p^{\gamma} n-p^{\gamma^{\prime}} p^{-\gamma}(n+1)\right|_{p}}=\delta_{\gamma \gamma^{\prime}} \\
\Omega\left(|m-n|_{p}\right)=\delta_{m n}, \quad m, n \in \frac{Q_{p}}{Z_{p}}
\end{gathered}
$$

Следствие доказано.

ЗАМЕЧАНИЕ. Из следствия вытекают следуюшие рекуррентные соотношения:

$$
\begin{gathered}
\lambda_{\gamma-1, p^{-1} n}-\lambda_{\gamma n}=p^{\gamma-1} T^{\left(\gamma-1, p^{-1} n\right)}-p^{\gamma-1} T^{(\gamma n)}, \\
T^{(\gamma n)}=T^{\left(\gamma-1, p^{-1} n\right)}+p^{1-\gamma}\left(\lambda_{\gamma n}-\lambda_{\gamma-1, p^{-1} n}\right) .
\end{gathered}
$$

В частности, последовательность $\left\{\lambda_{\gamma n}\right\}$ для фиксированного $n$ неотрицательна и не возрастает с ростом $\gamma$. 


\section{4. ПРИМЕРЫ ГЕНЕРАТОРОВ И СВОЙСТВА ДИФФУЗИИ}

ПримеР 1. Рассмотрим трансляционно-инвариантный случай. Тогда $T^{(\gamma n)}=T^{(\gamma)}$ и суммирование по $n$ в (7) дает

$$
\sum_{n} \Omega\left(\left|p^{\gamma} x-n\right|_{p}\right)=1
$$

Обозначая $T^{(\gamma)}=f\left(p^{\gamma}\right)$, получаем для ядра

$$
T(x, y)=\sum_{\gamma} T^{(\gamma)} \delta_{1,\left|p^{\gamma} x-p^{\gamma} y\right|_{p}}=f\left(|x-y|_{p}\right) .
$$

Следовательно, выбор

$$
T^{(\gamma)}=p^{-\gamma(1+\alpha)}
$$

соответствует оператору Владимирова, для которого ядро задается как

$$
T(x, y)=\frac{1}{|x-y|_{p}^{1+\alpha}}
$$

Формула (14) в случае (16) принимает вид

$$
\begin{aligned}
\lambda_{\gamma n} & =p^{\gamma} T^{(\gamma)}+\left(1-p^{-1}\right) \sum_{\gamma^{\prime}=\gamma+1}^{\infty} p^{\gamma^{\prime}} T^{\left(\gamma^{\prime}\right)}= \\
& =p^{\gamma} p^{-\gamma(1+\alpha)}+\left(1-p^{-1}\right) \sum_{\gamma^{\prime}=\gamma+1}^{\infty} p^{\gamma^{\prime}} p^{-\gamma^{\prime}(1+\alpha)}=p^{-\gamma \alpha} \frac{1-p^{-\alpha-1}}{1-p^{-\alpha}} .
\end{aligned}
$$

ПримеР 2. Рассмотрим случай

$$
T^{(\gamma n)}=f\left(p^{\gamma}\right) g\left(\left|p^{-\gamma} n-n_{0}\right|_{p}\right)
$$

Для ядра получим

$$
\begin{aligned}
T(x, y) & =\sum_{\gamma} f\left(p^{\gamma}\right) \delta_{1,\left|p^{\gamma} x-p^{\gamma} y\right|_{p}} \sum_{n} g\left(\left|p^{-\gamma} n-n_{0}\right|_{p}\right) \Omega\left(\left|p^{\gamma} x-n\right|_{p}\right)= \\
& =f\left(|x-y|_{p}\right) \sum_{\gamma} \delta_{p^{\gamma},|x-y|_{p}} \sum_{n} g\left(\left|p^{-\gamma} n-n_{0}\right|_{p}\right) \Omega\left(\left|p^{\gamma} x-n\right|_{p}\right) .
\end{aligned}
$$

Можно показать, что ряд

$$
\sum_{n \in Q_{p} / Z_{p}} g\left(\left|n-n_{0}\right|_{p}\right) \Omega\left(|x-n|_{p}\right)= \begin{cases}g\left(\left|x-n_{0}\right|_{p}\right) & \text { для }\left|x-n_{0}\right|_{p}>1 \\ g(0) & \text { для }\left|x-n_{0}\right|_{p} \leqslant 1\end{cases}
$$

т.е.

$\sum_{n \in Q_{p} / Z_{p}} g\left(\left|n-n_{0}\right|_{p}\right) \Omega\left(|x-n|_{p}\right)=g\left(\left|x-n_{0}\right|_{p}\right)\left(1-\Omega\left(\left|x-n_{0}\right|_{p}\right)\right)+g(0) \Omega\left(\left|x-n_{0}\right|_{p}\right)$. 
Отсюда следует, что

$$
\begin{aligned}
& \sum_{n \in Q_{p} / Z_{p}} g\left(\left|p^{-\gamma} n-n_{0}\right|_{p}\right) \Omega\left(\left|p^{\gamma} x-n\right|_{p}\right)= \\
& \quad=g\left(\left|x-n_{0}\right|_{p}\right)\left(1-\Omega\left(\left|p^{\gamma} x-n_{0}\right|_{p}\right)\right)+g(0) \Omega\left(\left|p^{\gamma} x-n_{0}\right|_{p}\right) .
\end{aligned}
$$

Это дает для ядра выражение

$T(x, y)=f\left(|x-y|_{p}\right)\left(g\left(\left|x-n_{0}\right|_{p}\right)\left(1-\Omega\left(|| x-\left.y\right|_{p} x-\left.n_{0}\right|_{p}\right)\right)+g(0) \Omega\left(|| x-\left.y\right|_{p} x-\left.n_{0}\right|_{p}\right)\right)$.

Из следствия к теореме 3 и формулы (18) следует выражение для собственных значений генератора, соответствуюшего ядру (19),

$$
\lambda_{\gamma n}=p^{\gamma} f\left(p^{\gamma}\right) g\left(\left|p^{-\gamma} n-n_{0}\right|_{p}\right)+\left(1-p^{-1}\right) g\left(\left|p^{-\gamma} n-n_{0}\right|_{p}\right) \sum_{\gamma^{\prime}=\gamma+1}^{\infty} p^{\gamma^{\prime}} f\left(p^{\gamma^{\prime}}\right) .
$$

Рассмотрим релаксацию характеристической функции единичного диска $\phi=\Omega\left(|x|_{p}\right)$ для ультраметрической диффузии с ядром (7). Характеристическая функция имеет следуюшее разложение по р-адическим всплескам (см. [2]):

$$
\begin{gathered}
\left\langle\Omega\left(|x|_{p}\right), \psi_{\gamma j n}\right\rangle=p^{-\gamma / 2} \theta(\gamma) \delta_{n 0}, \\
\theta(\gamma)= \begin{cases}0, & \gamma \leqslant 0 \\
1, & \gamma \geqslant 1,\end{cases} \\
\Omega\left(|x|_{p}\right)=\sum_{j=1}^{p-1} \sum_{\gamma=1}^{\infty} p^{-\gamma / 2} \psi_{\gamma j 0}(x) .
\end{gathered}
$$

Следовательно, оператор $T$ действует на $\Omega\left(|x|_{p}\right)$ следующим образом:

$$
T \Omega\left(|x|_{p}\right)=\sum_{j=1}^{p-1} \sum_{\gamma=1}^{\infty} p^{-\gamma / 2} \lambda_{\gamma 0} \psi_{\gamma j 0}
$$

Из формулы (14) для населенности единичного диска с центром в нуле получим

$$
\begin{aligned}
S(t) & =\left\langle\Omega\left(|x|_{p}\right), e^{-t T} \Omega\left(|x|_{p}\right)\right\rangle=(p-1) \sum_{\gamma=1}^{\infty} p^{-\gamma} e^{-t \lambda_{\gamma} 0}= \\
& =(p-1) \sum_{\gamma=1}^{\infty} p^{-\gamma} \exp \left[-t\left(p^{\gamma} T^{(\gamma 0)}+\left(1-p^{-1}\right) \sum_{\gamma^{\prime}=\gamma+1}^{\infty} p^{\gamma^{\prime}} T^{\left(\gamma^{\prime} 0\right)}\right)\right] .
\end{aligned}
$$

Аналогично

$$
\begin{aligned}
\left\langle\Omega\left(\left|p^{\gamma} x-n\right|_{p}\right), \psi_{\gamma^{\prime} j n^{\prime}}\right\rangle & =\left\langle\Omega\left(\left|p^{\gamma} x-n\right|_{p}\right), p^{-\gamma^{\prime} / 2} \chi\left(p^{\gamma^{\prime}-1} j x\right) \Omega\left(\left|p^{\gamma^{\prime}} x-n^{\prime}\right|_{p}\right)\right\rangle= \\
& =p^{-\gamma^{\prime} / 2} \theta\left(\gamma^{\prime}-\gamma\right) \chi\left(p^{\gamma^{\prime}-\gamma-1} j n\right) p^{\gamma} \Omega\left(\left|p^{\gamma^{\prime}-\gamma} n-n^{\prime}\right|_{p}\right),
\end{aligned}
$$


откуда следует, что

$$
\Omega\left(\left|p^{\gamma} x-n\right|_{p}\right)=p^{\gamma} \sum_{j=1}^{p-1} \sum_{\gamma^{\prime}=\gamma+1}^{\infty} p^{-\gamma^{\prime} / 2} \chi\left(p^{\gamma^{\prime}-\gamma-1} j n\right) \psi_{\gamma^{\prime} j p^{\gamma^{\prime}-\gamma} n}(x),
$$

где была использована формула

$$
\Omega\left(\left|p^{\gamma^{\prime}-\gamma} n-n^{\prime}\right|_{p}\right)=\delta_{p^{\gamma^{\prime}-\gamma} n, n^{\prime}}
$$

Формула (21) позволяет получить аналоги соотношения (20) для смещенных дисков.

Отметим, что $p^{\gamma^{\prime}-\gamma} n=0$ в $Q_{p} / Z_{p}$, когда $\gamma^{\prime}$ достаточно велико. Поэтому ряд по всплескам в (21) содержит конечное число всплесков с третьим индексом, не равным нулю. Следовательно, асимптотическая зависимость от времени для выражения

$$
\left\langle\Omega\left(\left|p^{\gamma} x-n\right|_{p}\right), e^{-t T} \Omega\left(\left|p^{\gamma^{\prime}} x-n^{\prime}\right|_{p}\right)\right\rangle
$$

совпадает с асимптотикой для (20).

\section{5. ЗАКЛЮЧЕНИЕ}

Подход, развиваемый в данной работе, может быть применен к проблеме исследования диффузии на иерархических ландшафтах энергии. Концепция иерархических ландшаф̆тов энергии привлекает большое внимание в связи с изучением сложных систем, в частности, стекол, кластеров и белков [17]-[22], [26]-[29]. Обсудим эту концепцию на языке "кинетики из бассейна в бассейн" [22]. В рамках данной концепшии предполагается, что сложная система имеет большое число метастабильных конфигураций (состояний), соответствуюших локальным минимумам энергии. Локальные минимумы кластеризованы в иерархически вложенные бассейны минимумов, а именно каждый большой бассейн состоит из меньших бассейнов, каждый из которых состоит из еше меньших, и так далее. Другими словами, иерархия бассейнов обладает ультраметрической геометрией. Кроме того, бассейны минимумов отделены один от другого иерархически устроенным набором барьеров. Это значит, что высокие барьеры разделяют большие бассейны (соответственно вероятность перехода между точками больших бассейнов мала), а меньшие бассейны (внутри больших) разделены меньшими барьерами (и соответствуюшая вероятность перехода из точки в точку выше).

Концепция иерархических ландшаф̆тов энергии может рассматриваться как разновидность приближения сложного ландшафта энергии иерархическим. Динамику системы с иерархическим ландшафтом энергии естественно описывать моделью ультраметрической диффузии. Сушествует потребность в аналитических методах для описания иерархических ландшафтов энергии, явлений релаксации в сложных системах и "кинетики из бассейна в бассейн" (см. [18]-[22]). Настоящая работа посвящена разработке именно таких методов. Мы показали, что для некоторого широкого класса ультраметрических диффузий соответствуюшие генераторы ( $p$-адические псевдодифференциальные операторы) могут быть диагонализованы в базисе $p$-адических всплесков, введенном в работе [2]. Это означает, что случайное блуждание на соответствуюшем ландшаф̆те энергии может быть изучено аналитически. 
Благодарности. Автор благодарит В. С. Владимирова, В. А. Аветисова, И. В. Воловича, А.Ю. Хренникова, А.Н. Кочубея, А.Х. Бикулова и В.А. Осипова за обсуждения и важные замечания. Работа частично поддержана фондом INTAS YSF 2002-160 F2, CRDF (грант № UM1-2421-KV-02), Российским фондом фундаментальных исследований (грант № 02-01-01084) и грантом на поддержку научной школы НШІ № 1542.2003 .1 .

\section{Список литературы}

[1] В. С. Владимиров, И. В. Волович, Е. И. Зеленов. p-Адический анализ и математическая физика. М.: Наука, 1994.

[2] С. В. Козырев. Изв. РАН. Сер. матем. 2002. Т. 66. № 2. С. 149; math-ph/0012019.

[3] S. Albeverio, W. Karowowsky. A random walk on p-adic numbers. In: Stochastic Process-Physics and Geometry II. Proc. Locarno (1991). Eds. S. Albeverio, U. Cattaneo, D. Merlini. Singapore: World Scientific, 1995. P. 61.

[4] А. Н. Кочубей. ТМФ. 1993. Т. 96. № 1. С. 123.

[5] А. Н. Кочубей. Изв. РАН. Серия матем. 1991. Т. 55. № 6. С. 1312.

[6] A. N. Kochubei. Pseudodifferential Equations and Stochastics over Non-archimedean Fields. New York: Marcel Dekker, 2001.

[7] V.A. Avetisov, A.H. Bikulov, S. V. Kozyrev. J. Phys. A. 1999. V. 32. P. 8785; cond-mat $/ 9904360$.

[8] V. A. Avetisov, A. H. Bikulov, S. V. Kozyrev, V. A. Osipov. J. Phys. A. 2002. V. 35. P. 177; cond-mat/0106506.

[9] V.A. Avetisov, A.Kh. Bikulov, V.A. Osipov. p-Adic description of characteristic relaxation in complex systems. Submitted to J. Phys. A; cond-mat/0210447.

[10] I. V. Volovich. Class. Q. Grav. 1987. V. 4. P. L83.

[11] A. Khrennikov. p-Adic Valued Distributions in Mathematical Physics. Dordrecht: Kluwer, 1994.

[12] A. Khrennikov. Non-Archimedean Analysis: Quantum Paradoxes, Dynamical Systems and Biological Models. Math. and its Appl. V. 427. Dordrecht: Kluwer, 1997.

[13] G. S. Djordjevic, B. Dragovich. Mod. Phys. Lett. A. 1997. V. 12. P. 1455.

[14] G. Parisi, N. Sourlas. Eur. J. Phys. B. 2000. V. 14. P. 535; cond-mat/9906095.

[15] D. M. Carlucci, C. De Dominicis. On the replica Fourier transform. cond-mat/9709200.

[16] C. De Dominicis, D. M. Carlucci, T. Temesvari. J. Phys. I France. 1997. V. 7. P. 105; cond-mat/9703132.

[17] M. Mezard, G. Parisi, M. Virasoro. Spin-Glass Theory and Beyond. Singapore: World Scientific, 1987.

[18] H. Frauenfelder. Nature Struct. Biol. 1995. V. 2. P. 821.

[19] D. Th. Leeson, D. A. Wiersma. Nature Struct. Biol. 1995. V. 2. P. 848.

[20] D. Sherrington. Physica D. 1997. V. 107. P. 117.

[21] H. Frauenfelder, D. Th. Leeson. Nature Struct. Biol. 1998. V. 5. P. 757.

[22] O. M. Becker, M. Karplus. J. Chem. Phys. 1997. V. 106. P. 1495.

[23] J. P. Serre. Trees. New York, Berlin: Springer, 1980.

[24] A. T. Ogielski, D. L. Stein. Phys. Rev. Lett. 1985. V. 55. № 15. P. 1634.

[25] Hajime Yoshino. Hierarchical diffusion, aging and multifractality. cond-mat/9604033.

[26] M. Mezard, G. Parisi, N. Sourlas, G. Toulouse, M. Virasoro. Phys. Rev. Lett. 1984. V. 52. P. 1156

[27] K. Binder, A. P. Young. Rev. Mod. Phys. 1986. V. 58. P. 801.

[28] A. Ansary, J. Berendzen, S.F. Bowne, H. Frauenfelder, I. E. T. Iben, T. B. Sauke, E. Shyamsunder, R. D. Young. Proc. Natl. Acad. Sci. USA. 1985. V. 82. P. 5000.

[29] H. Frauenfelder, S. G. Sligar, P. G. Wolynes. Science. 1991. V. 254. P. 1598. 